\title{
The effect of material mixing on heat transfer in the process of contact drying
}

\author{
Jan Havlík ${ }^{1, *}$, Tomáš Dlouhý ${ }^{1}$ \\ ${ }^{1}$ Czech Technical University in Prague, Faculty of Mechanical Engineering, Department of Energy Engineering, Technická 4, 16607 \\ Prague, Czech Republic
}

\begin{abstract}
This paper deals with the process of heat transfer in contact drying. Heat transfer during contact drying for a stagnant bed without mixing and drying with a mechanically agitated bed were theoretically and experimentally investigated for a contact paddle dryer. The effect of material mixing on the process of heat transfer was evaluated. For a theoretical description of heat transfer in a contact dryer, the penetration model was used. Experiments with green wood chips were realized on an experimental paddle dryer. In both cases, the stagnant bed and the mechanically agitated bed, the theoretical values were slightly higher compared to the experimental results. Mixing intensifies the process, it showed an increase to roughly twice in heat transfer for the tested experimental dryer.
\end{abstract}

\section{Introduction}

Indirect (contact) dryers have been successfully used to dry a wide variety of materials ranging from food and dairy products over energy fuel as coal and biofuel to chemical and other miscellaneous products such as carbon black, colloidal clay, pigments, peat and many kinds of sludge [1]. In indirect dryers, the material and the heating medium are separated by a heat transfer surface. The heat is fed to the dried material through this surface, which defines the drying space. The heating medium is usually steam or hot water. Wet material is dried by coming into contact with the heated surface; thus, heat transfer to the wet material is mainly through conduction from this surface [1]. An advantage and energy efficiency of indirect drying into energy systems can be found in [2]. Although, the theoretical basis of contact drying is established and available in the literature, there is a need for stable and reliable models to quantify and predict drying rates and drying times based on the experiential data to achieve satisfactory accuracy for designing and optimizing drying processes in a wide range of design and operating parameters [1].

\section{Heat transfer in contact drying}

The heat transfer from a hot surface to free flowing and mechanically agitated particulate dry material and the subsequent problem of heat transfer to stirred beds during contact drying has been investigated by Schlünder and co-workers [3-6]. In both cases the socalled penetration model was applied to describe mechanical mixing [5].

\subsection{Heat transfer from the heated wall to the packed bed}

Application of the penetration model is well described in [7] and [8]. In Fig. 1, the scheme of heat transfer from the heated wall to the particulate material is shown. There is a steep temperature drop in the immediate vicinity of the wall, $\vartheta_{w}-\vartheta_{0}$, followed by a further decrease toward the interior of the bed, $\vartheta_{0}-\vartheta_{\text {bed }}$ (average caloric bed temperature).

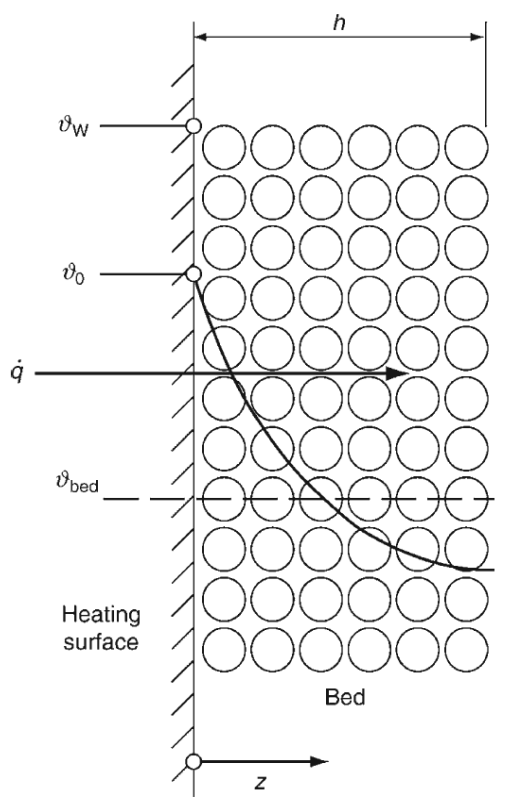

Fig. 1. Scheme of heat transfer from a wall to an adjoining bed of particles [6].

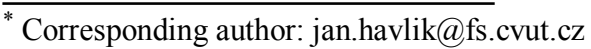


The temperature jump $\vartheta_{w}-\vartheta_{0}$, and the temperature difference $\vartheta_{0}-\vartheta_{\text {bed }}$ are assigned to contact heat resistance, $1 / \alpha_{w s}$, and to penetration resistance, $1 / \alpha_{b e d}$, respectively. Therefore, it is

$$
\begin{gathered}
\alpha_{w s}=\dot{q} /\left(\vartheta_{w}-\vartheta_{0}\right) \\
\alpha_{b e d}=\dot{q} /\left(\vartheta_{0}-\vartheta_{\text {bed }}\right)
\end{gathered}
$$

The overall heat transfer coefficient (HTC) $\alpha$ is

$$
\alpha=\dot{q} /\left(\vartheta_{w}-\vartheta_{b e d}\right)
$$

and

$$
\frac{1}{\alpha}=\frac{1}{\alpha_{w s}}+\frac{1}{\alpha_{b e d}}
$$

Using the standard boundary condition of constant temperature $\vartheta_{0}$, the time-averaged heat penetration coefficient of the stagnant bed $\alpha_{b e d, d r y}$ is obtained to

$$
\alpha_{\text {bed,dry }}=\frac{2}{\sqrt{\pi}} \frac{\sqrt{(\rho \cdot c \cdot \lambda)_{b e d, d r y}}}{\sqrt{t_{R}}}=\alpha_{b e d}
$$

Here, $\rho, c, \lambda$ are respectively the density, heat capacity at constant pressure and the thermal conductivity, $t_{R}$ is the residence time of the bed on the heating surface. The contact HTC between the wall and the bed $\alpha_{W S}$ is obtained to

$$
\alpha_{W S}=\varphi \alpha_{W P}+\alpha_{r a d}
$$

where $\varphi$ is the surface coverage factor, $\alpha_{W P}$ is the wallparticle HTC, $\alpha_{\text {rad }}$ is the HTC by radiation.

$$
\alpha_{W P}=\frac{4 \lambda_{g}}{d}\left[\left(1+\frac{2(l+d)}{d}\right) \ln \left(1+\frac{d}{2(l+\delta)}\right)-1\right]
$$

where $l$ is the modified mean free path of gas molecules, $\lambda_{g}$ is the thermal conductivity of the gas, $d$ is the particle diameter, $\delta$ is the surface roughness of the particles.

$$
\alpha_{\text {rad }}=4 C_{W, b e d} T^{3}
$$

where $T$ is the absolute temperature and the overall radiation exchange coefficient $C_{W \text {,cbed }}$ is calculated as

$$
C_{W, \text { cbed }}=\sigma /\left(1 / \varepsilon_{W}+1 / \varepsilon_{\text {bed }}-1\right)
$$

where $\varepsilon_{W}, \varepsilon_{\text {bed }}$ are the emission coefficients of wall and bed, respectively. The description of calculation and the determination of the parameters above are described in detail in [7].

\subsection{Effect of mixing}

For contact drying, the mechanical mixing is described in the penetration model $[4,5,8]$. To obtain the similar expressions as for a stirred bed it is at first assumed that there exists a certain time period $t_{R}$ during which the bed is considered dense and the heat transfer to the bed is governed by Equation (5). After the time period $t_{R}$ a perfect mixing of the bed is achieved with more intensive heat transfer depending on the method and frequency of mixing. This assumption results in oscillations of instantaneous HTC which is difficult to describe. For practical use the time average of these values is evaluated in a similar way to the overall HTC of the stirred bed. The value of $t_{R}$ is calculated from a simple relation

$$
t_{R}=N_{\text {mix }} \cdot t_{\text {mix }}
$$

where $\mathrm{N}_{\text {mix }}$ is the mixing number, which is an experimentally determined parameter dependent only on the mechanical properties of the system as well as on the Froude number $\mathrm{Fr}$. The mixing number lies roughly in the wide range of 2-25 [8] and is rather independent of the hot surface temperature, operating pressure, or the moisture content in the dried material. It can be simply imagined as the number of revolutions of the stirrer required to reach a perfectly mixed bed of particles. As a rough estimation, in [4] the following correlation is recommended for the prediction of the mixing number

$$
N_{\text {mix }}=C \cdot F r^{x}
$$

where $F r=(2 \pi n)^{2} D / 2 g, D$ is the diameter of the disc or the rotary drum and $n$ is the number of revolutions of the mixing device. The values of $C$ and $x$ correspond with various types of dryers. Finally, $t_{\text {mix }}$ is the time constant of the stirrer, which may be taken as the time required for one revolution of the stirrer.

\subsection{Effect of drying}

In [4], the penetration model has been extended to contact drying, where it is assumed that a steep drying front propagates into the bed during the time period $t_{R}$. Not only the temperature, but also the moisture profile is changed at the end of each period. The contact HTC $\alpha_{W S}$ does not change in principle [6]. On the contrary, the consumption of heat by evaporation in the interior of the bed (the latent heat sink) has a significant influence on the penetration coefficient $\zeta$ which in this case is obtained to

$$
\begin{gathered}
\alpha_{b e d}=\frac{2}{\sqrt{\pi}} \frac{\sqrt{\rho \cdot c \cdot \lambda}}{\sqrt{t_{R}}} \frac{1}{e r f \zeta}=\frac{\alpha_{\text {bed,dry }}}{\operatorname{erf} \zeta} \\
\sqrt{\pi} \zeta \exp \left(\zeta^{2}\right)\left[\left(\frac{\alpha_{w s}}{\alpha_{d r y}}-1\right) \operatorname{erf} \zeta+1\right]=\frac{1}{P h}\left(\frac{\alpha_{w s}}{\alpha_{d r y}}-1\right)
\end{gathered}
$$

The phase change number $P h$ is

$$
P h=\frac{X \cdot \Delta h_{v}}{c_{b e d, d r y} \cdot\left(\vartheta_{w}-\vartheta_{b e d}\right)}
$$

where $X$ is the material moisture content, $\Delta h_{v}$ is the evaporation heat.

\section{Experiment}

The indirect drum dryer (see Fig. 2) in laboratory scale was designed and manufactured for testing the drying method and for increasing understanding about the 
indirect drying process. The dryer was designed with an electric heated shell coated with insulation. The dryer has a cylindrical shape with a diameter of $0.26 \mathrm{~m}$ and a length of $1 \mathrm{~m}$. The dryer can be equipped with a paddlewheel agitator with a rotation velocity of $9 \mathrm{rpm}$ in order to intensify the heat transfer process. The dryer works in batch mode. In the course of the experiments the dryer is closed and sealed to permit air penetration with the aim to reduce the energy consumption of the drying.

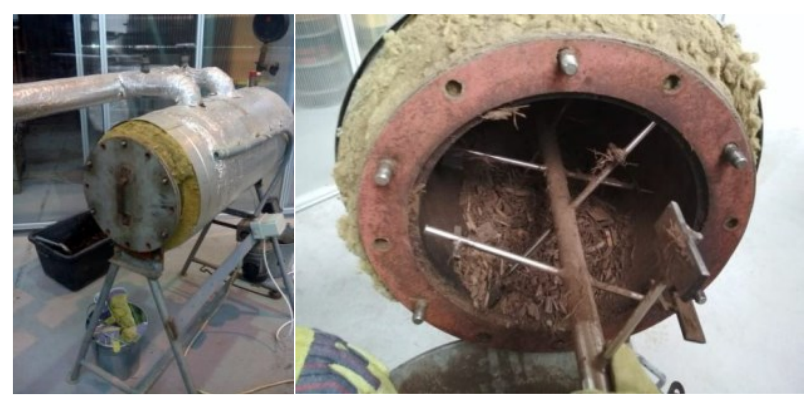

Fig. 2. Experimental indirect dryer

Two modes of dryer operation were tested, drying in a stagnant bed without mixing and drying with a mechanically agitated bed. The measured parameters are the temperature of the heated dryer surface, the pressure inside the dryer, the released vapour temperature, the input and output weight of the dried material, the input and output moisture content in the dried material and the electricity consumption for heating.

The tested type of biomass was green wood chips, which was bought from an external supplier and stored in an outdoor open area (see Fig. 3), so the inlet water content was from 55 to $66 \%$. This material is very inhomogeneous with a high moisture content.

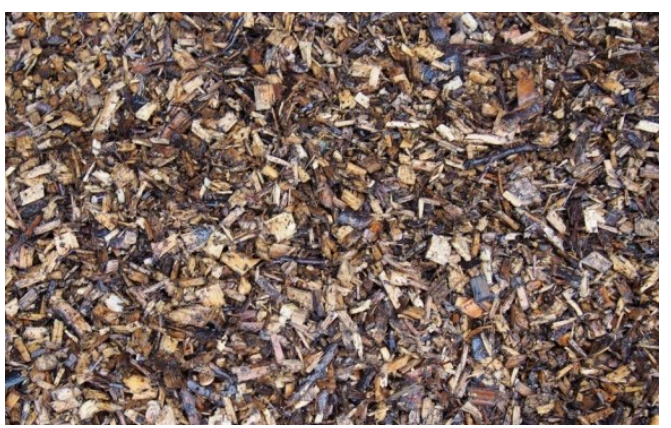

Fig. 3. Tested material - green wood chips

\subsection{Experimental evaluation}

The aim of the experiments was to determine the overall HTC $\alpha_{\text {exp }}$ between the wall and the heating material, which is calculated from measured parameters as

$$
\alpha_{\text {exp }}=\frac{Q}{A \cdot \Delta t_{l o g}}
$$

where $A$ is the heat exchange surface covered by the material and $\Delta t_{l o g}$ is the temperature difference between the heating surface and the dried material. The heat to evaporate water $Q$ is calculated as

$$
Q=M_{e w} \cdot \Delta h_{v}
$$

where $M_{e w}$ is the amount of evaporated water. The heat exchange surface scattered with the material (see Fig. 2 the right part) is calculated as

$$
\mathrm{A}=s \cdot L
$$

where $s$ is the part of the circuit where the drying material lies and $L$ is the length of the dryer.

\section{Results}

Green wood chips with a moisture content (MC) around $60 \%$ were used for drying tests. For all experiments, the dryer was heated to a temperature of $140^{\circ} \mathrm{C}$. The dryer is designed in atmospheric solution thus it is open to the atmosphere for releasing the evaporated vapor. So the dryer works with a small overpressure to the ambient atmosphere (about 10 to $20 \mathrm{~Pa}$ ).

The effect of the drum filling was investigated for $11 \%, 13 \%$ and $21 \%$ of the drum volume. In Table 1 , a comparison of the overall HTC calculated by the theoretical model described above with values obtained from the experimental results is shown. Approximate values were used taken from the recommendations of the literature for the model coefficients. Determining mixing number $\mathrm{N}_{\text {mix }}$ is the key problem for the use of the model. In the case $\mathrm{N}_{\text {mix }}-1$ (In Table 1, theory 1 for Agitated bed), the value 12 is chosen from the recommended range $2-25$ [7]. In the case $\mathrm{N}_{\text {mix }}-2$ (In Table 1, theory 2 ), the equation (14) derived experimentally for one specific stirrer and dryer [7] is used. The evaluation does not take into account the phase of material heating and focuses only on the part pertaining to the evaporation of water from the material.

Table 1. Comparison of the theoretical and experimental results of the overall HTC

\begin{tabular}{|l|l|c|c|c|c|c|c|c|}
\hline \multicolumn{2}{|c|}{} & \multicolumn{3}{c|}{ Stagnant bed } & \multicolumn{5}{c|}{ Agitated bed } \\
\hline Drying time & $\mathrm{h}$ & 1 & 1 & 2 & 1 & 1 & 1 & 2 \\
\hline Drum filling & $\%$ & 11 & 21 & 13 & 21 & 11 & 13 & 13 \\
\hline $\mathrm{MC}$ - inlet & $\%$ & 52 & 60 & 59 & 58 & 55 & 59 & 59 \\
\hline MC - outlet & $\%$ & 43 & 49 & 39 & 40 & 44 & 49 & 32 \\
\hline Results & \multicolumn{7}{|l|}{} \\
\hline$\alpha$ - experiment & $\mathrm{W} / \mathrm{m}^{2} \mathrm{~K}$ & 32 & 37 & 37 & 70 & 64 & 70 & 59 \\
\hline$\alpha$ - theory 1 & $\mathrm{W} / \mathrm{m}^{2} \mathrm{~K}$ & 45 & 48 & 36 & 93 & 93 & 94 & 92 \\
\hline$\alpha$ - theory 2 & $\mathrm{W} / \mathrm{m}^{2} \mathrm{~K}$ & - & - & - & 100 & 100 & 100 & 100 \\
\hline difference & $\%$ & -29 & -23 & 2 & -24 & -31 & -25 & -36 \\
\hline
\end{tabular}


The experimental results of the HTC are approximately $30 \%$ less than the theoretically calculated results. In the experiments, the dependence of values of the HTC on the material moisture content was apparent. The effect of mixing is evident in the good agreement for the theoretical and experimental results, the increase in the heat transfer is roughly twice as much for the tested dryer.

\section{Conclusion}

The heat transfer during contact drying for a stagnant bed without mixing and drying with a mechanically agitated bed was theoretically and experimentally investigated for a contact paddle dryer. Approximate values were used taken from the recommendations of the literature for the model coefficients. The lack of certainty in defining all the parameters of the mixing process suggests the need to verify the value of the mixing number experimentally for each specific case. However, it is evident that the method used for mixing has a major influence on the intensification of the drying process; the increase of heat transfer roughly twice was reached by mixing for the tested case.

The next steps in the research will be focused on optimizing the geometry of the stirrer and on extending the experiments for other input and operating conditions and drying material properties. It will make it possible to determine the stirrer characteristics in wider ranges and to describe the drying process in various conditions.

This work was supported by the Ministry of Education, Youth and Sports under OP RDE grant number CZ.02.1.01/0.0/0.0/16_019/0000753 "Research centre for lowcarbon energy technologies" and by Technology Agency of the Czech Republic, project No. TH03010395.

\section{References}

1. M. Intelvi, A. Picado, J. Martínez. WASET 5, 199 (2011)

2. J. Havlik, T. Dlouhy, JCHEJ 50, 792-798 (2017)

3. E. U. Schlünder, Chem. Eng. Process. 18, 31-53 (1984)

4. E. U. Schlünder and N. Mollekopf, Chem. Eng. Process. 18, 93-111 (1984)

5. E. Tsotsas, E.U. Schlünder. Chem. Eng. Process. 20, 277-285 (1986)

6. E. Tsotsas, E.U. Schlünder. Chem. Eng. Process. 21, 199-208 (1987)

7. VDI heat atlas (Springer, New York, 2010)

8. A. S. Mujumdar. Handbook of Industrial Drying (Marcel Dekker, New York, 1990) 